Background IL10 is a pleiotrophic cytokine that inhibits the antigen specific activation of Th1 clones by reducing both the antigen presenting capacity and the production of pro-inflammatory cytokines by monocytes. It is also a potent activation and differentiation factor for B lymphocytes. SLE patients show increased IL10 serum levels associated with the pathogenesis of the disease. Peripheral blood mononuclear cells from SLE patients, and their relatives, constitutively produce high levels of the cytokine, suggesting an hereditary component in IL10 production. AntiIL10 antibodies administration to SLE patients may be beneficial in the management of refractory cases. The IL10 gene promoter contains two dinucleotide repeats (microsatellites): IL10G and IL10R, close to transcription factor binding sites, probably playing an important role in the transcription regulation.

Objectives To evaluate the association of the IL10G microsatellite with SLE in Spanish patients.

Methods 80 unrelated SLE patients from Canary Islands (Spain) fulfilling at least four of the ACR criteria and 79 sex-matched healthy donors were included. A segment of the IL10 gene containing the microsatellite was amplified by PCR and the resulting products were electrophoresed in denaturing polyacrilamide gels. Chi-square test was used for statistical analysis.

Results No particular IL10G allele was found to be significantly associated with SLE. Neverthaless, we found an elevated risk of serositis in carriers of the allele 19 ( $\mathrm{p}=0.007)$, a significant association of the $19 / 23$ genotype with seizures $(p=0.018)$, and a reduced risk of renal disorder $(\mathrm{p}=0.047)$ and thrombocytopenia $(\mathrm{p}=0.049)$ in carriers of the allele 23 .

Conclusion Although IL10G alleles seem not to influence susceptibility to SLE in Spaniards, some of them may be related to the presence of several clinical manifestations of the disease.

\section{FRI0090 THE PRESENCE OF MICROCHIMERISM IN SYSTEMIC LUPUS ERYTHEMATOSUS: PRELIMINARY DATA}

M Mosca, M Curcio, S Lapi, R Neri, S Italia, D Totti, G Rizzo, S Bombardieri. Rheumatology Unit, University of Pisa, Pisa, Italy

10.1136/annrheumdis-2001.1232

Background The development of microchimerism after solid organ transplantation, but also after blood transfusions and during pregnancy, is no longer considered controversial. Controversy still exists on the immunological significance of this phenomenon, such for example its role on the development of tolerance to the graft, or of some autoimmune conditions such as scleroderma (SSc). In this respect several studies have showed the presence of fetal cells not only in the peripheral blood but also in the skin of SSc patients. These observations, along with epidemiological (female prevalence, onset after childbearing years) and clinical (similarities with chronic graft versus host disease) data, have led to the hypothesis that microchimerism may play a role in the aetiopathogenesis of this disease.

Objectives The aim of our study was to evaluate the prevalence of microchimerism in a population of SLE patients in comparison with SSc patients followed at our Unit.

Methods Anticoagulated peripheral blood was obtained from 22 female patients (13 SLE and 9 SSc) who had a previous male pregnancy followed at our Unit. Genomic DNA was isolated from anticoagulated blood using a DNA extraction kit. A specific Y-chromosome sequence was detected by amplifying DNA in a nested-polymerase chain reaction (PCR) using published primers.
Results Microchimerism was present in $62 \%$ of SLE patients and $89 \%$ of patients with SSc, the mean time interval between the pregnancy and the analysis was of 27 years and no significative differences were observed between positive and negative patients. In all scleroderma patients the pregnancy occurred before the onset of the disease (onset of Raynaud's phenomenon). In SLE patients 38\% of the pregnancies occurred before the onset of the disease, and $62 \%$ after the onset of the disease, however no differences in the development of microchimerism were observed.

Conclusion Since the literature data report a prevalence of 33\% of microchimerism in healthy controls, these preliminary data may suggest that microchimerism develops more easily in patients with various autoimmune conditions. Further studies on extended populations of CTD patients and healthy controls appear necessary, in order to look for permissive factors for its development, such for example immunosuppressive therapy or immunological abnormalities.

\section{REFERENCES}

1 Evans PC, et al. Blood 1999:93:2033-7

2 Nelson JL. Lupus 1999;8:370-4

3 Nelson JL. Curr Opin Rheumatol. 1998;10:564-71

\section{FRI0091 ANGIOGENESIS IN SYSTEMIC LUPUS ERYTHEMATOSUS (SLE) AND PRIMARY ANTIPHOSPHOLIPID SYNDROME (PAPS)}

${ }^{1} \mathrm{C}$ Navarro, ${ }^{2} \mathrm{~L}$ Candia-Zúníiga, ${ }^{2} \mathrm{LH}$ Silveira, ${ }^{1} \mathrm{~V}$ Ruiz, ${ }^{2} \mathrm{MC}$ Amigo. ${ }^{1} M o l e c u l a r$ Biology, Instituto Nacional de Enfermedades Respiratorias, Sección XVI, Mexico; ${ }^{2}$ Rheumatoly Service, Instituto Nacional de Cardiología, Sección XVI, Mexico

\subsection{6/annrheumdis-2001.1233}

Background Angiogenesis is an essential process of the body's physiology and plays a role in several diseases such as rheumatoid arthritis, neoplasias or atherosclerosis. Vascular endothelial growth factor (VEGF) and angiogenin (Ang) are factors that promote angiogenesis in vitro and in vivo. However, new vessels formation seems limited to tissues with some degree of inflammation or with hypoxia. SLE and PAPS are characterised by endothelial damage and the mechanisms of angiogenesis have not been explored in these diseases.

Objectives In this study we determine the possible role of VEGF and Ang in the pathogenesis of SLE and PAPS.

Methods We included 28 SLE patients, 10 PAPS patients and 24 matched controls. VEGF and Ang plasma levels were measured by ELISA.

Results The VEGF plasma levels were significantly increased in SLE patients compared with PAPS patients and controls, $\mathrm{p}<$ 0.01 . The Ang levels were similar in the three groups. No correlation between VEGF levels and clinical manifestations was found.

\begin{tabular}{llll}
\multicolumn{1}{l}{ Abstract FRI0091 Table 1 } \\
\hline Gender (F/M) & Age (years) & $\begin{array}{l}\text { VEGF (pg/ml) } \\
\text { (median/range) }\end{array}$ \\
\hline SLE Group & $24 / 4$ & $36.6 \pm 16.1$ & $70.25(4.69-341.2)$ \\
PAPS Group & $8 / 2$ & $36.2 \pm 8.0$ & $34.1(6.91-83.78)$ \\
Control & $19 / 5$ & $29.2 \pm 8.5$ & $23.48(0-307.4)$ \\
\hline
\end{tabular}

